\title{
Probe diagnostics of plasma in an electron cyclotron resonance ion source with a magnetic field generated by permanent magnets
}

\author{
Member Satoshi Ichimura (Hitachi Ltd) \\ Member Tadashi Sato (Hitachi Ltd) \\ Non-member Shoutaro Ooishi (Hitachi Ltd)
}

\begin{abstract}
An electron cyclotron resonance (ECR) ion source for a large area uniform ion beam with multipolar magnetic field is being developed. Hot electrons heated at the localized ECR region in the source ionize a large volume of gas in the discharge chamber, where a large area plasma is generated and the plasma loss is suppressed with the multipolar magnetic field. The electron energy and current distributions are measured with Langmuir probes to confirm the above plasma generation scheme.
\end{abstract}

Keywords: ECR, plasma, ion source, permanent magnet, multipolar magnetic field

\section{Introduction}

Recently there have been growing demands for reactive ion beams to microfabricate thin film devices. ECR discharge ion sources have no hot cathodes and are suitable for generating reactive ion beams for stable operation under chemically reactive gases. Permanent magnets have been used to form a resonance magnetic field for ECR discharge and/or a multipolar magnetic field for the confinement of plasma in many types of ion sources (1)-(4). These ion sources were proposed to get a uniform large area plasma by improving the antenna configuration and arrangements for moderate microwave power distribution.

By contrast, our ECR discharge ion source, presented here, introduces microwave power at a local point in the discharge chamber to produce hot electrons. Then, these hot electrons generate a uniform large area plasma in the magnetic field-free region surrounded by a multipolar magnetic field. The plasma characteristics of this ion source are presented in this paper.

\section{Conceptual Source Design}

As shown in Fig. 1, ECR discharge in a multipolar magnetic field occurs at first inside the strong magnetic field lines near the poles of the permanent magnets. Depending on the increase of gas pressure or microwave power, discharge plasma becomes larger along the longer magnetic field lines. Finally, the main plasma is generated in the magnetic field-free region.

The above phenomena are interpreted as follows. ECR discharge occurs in the region where electrons oscillate with better plasma confinement and shorter magnetic field lines. Depending on the increase of gas pressure or microwave power, the plasma is generated in the magnetic field-free region, after generation in the strong magnetic field region.

Plasma generation in the magnetic field-free region has many advantages such as low ion temperature, uniform electron density distribution and effective ion extraction, but often needs too large a gas pressure or microwave power. The following points describe conditions to form the plasma in the magnetic field-free region under moderate gas pressure or microwave power.

(1) The introduction of a high electric field intensity microwave to the region where microwave-heated hot electrons are generated.

(2) The production of a large volume, magnetic field-free region.

(3) The application of an electric field barrier to hot electrons to keep from the extraction electrode.

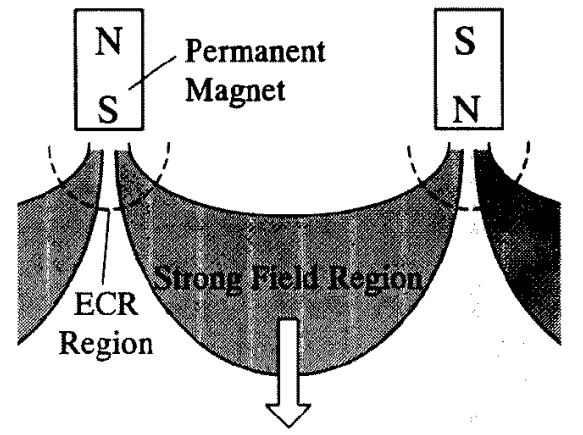

Field-Free Region

Fig.1. Plasma generation in ECR ion source with multipolar magnetic field.

\section{Experimental Arrangements}

For this experiment, an ECR ion source as shown in Fig.2 was constructed. Fig.2(a) shows a cross-sectional view of the experimental arrangements with the ion source, and (b) shows a front view of the ion source. Fig. 3 shows the calculated results of the two-dimensional magnetic field distribution. The vacuum chamber had a volume of $500 \times 500 \times 800 \mathrm{~mm}^{3}$ and was evacuated by a $550 \mathrm{l} / \mathrm{s}$ turbo-pump. The ECR ion source, whose discharge chamber had the dimensions of $360 \times 272 \times 170 \mathrm{~mm}^{3}$, was placed in the vacuum chamber. The discharge chamber wall was grounded like the vacuum chamber. The 
discharge chamber gas pressure was adjusted through the mass flow controller. The rectangular microwave guide $(96 \times 27 \mathrm{~mm})$ was used to introduce the microwave power into the chamber. Microwave power was matched with a three-stub tuner. The permanent magnets were mounted around the microwave guide. At the silica glass surface inside the microwave guide, the magnetic field strength was over $87.5 \mathrm{mT}$ (ECR condition magnetic field). At the same time, the field strength at the discharge chamber center was small, being below $3 \mathrm{mT}$ (Fig.3). So, the magnetic field strength decreased sharply from the silica glass surface to the discharge chamber center. Thus, a wide magnetic field-free area could be realized. To suppress plasma loss at the wall, a multipolar magnetic field was applied. The wall material was mild steel. The same size Sm-Co permanent magnets $(6 \times 20 \times 12 \mathrm{~mm}$; $12 \mathrm{~mm}$ magnetized direction) were used. Three columns of permanent magnets were mounted along the longitudinal direction of the microwave guide to get a magnetic field of over $87.5 \mathrm{~m}$ T at the silica glass surface. In the microwave guide, a certain volume, where it is difficult for electrons to diverge to the magnetic field-free region, was filled with silica glass in order to supply microwave power effectively to the plasma.

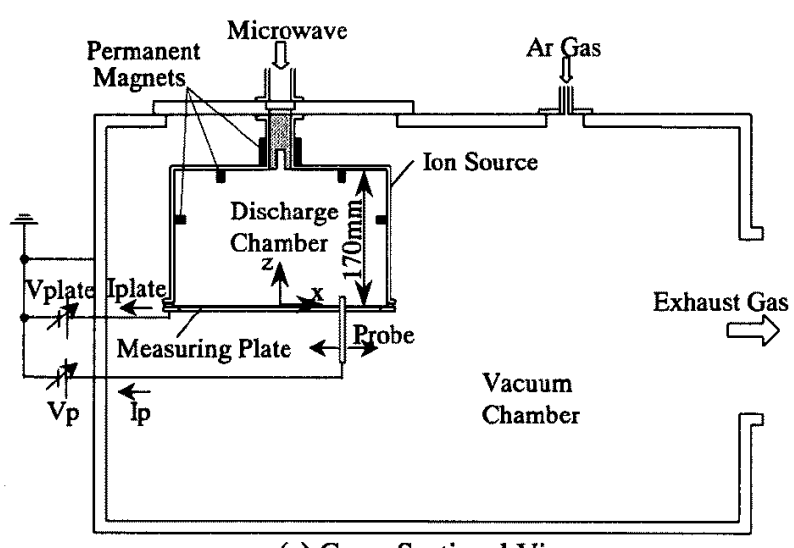

(a) Cross-Sectional View

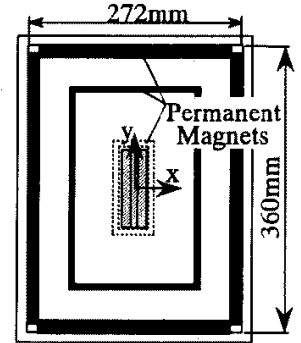

(b) Ion Source Front View

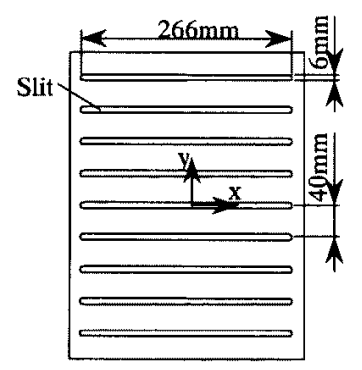

(c) Measuring Plate Front View
Fig.2. Experimental arrangements

On the beam extraction side, a measuring plate (Fig.2(c)) was mounted and plasma parameters were measured through its slits. The measuring plate was negatively biased to confine hot electrons and allow total ion current flowing into the plate to be measured.

In this study, the discharge chamber was set inside the

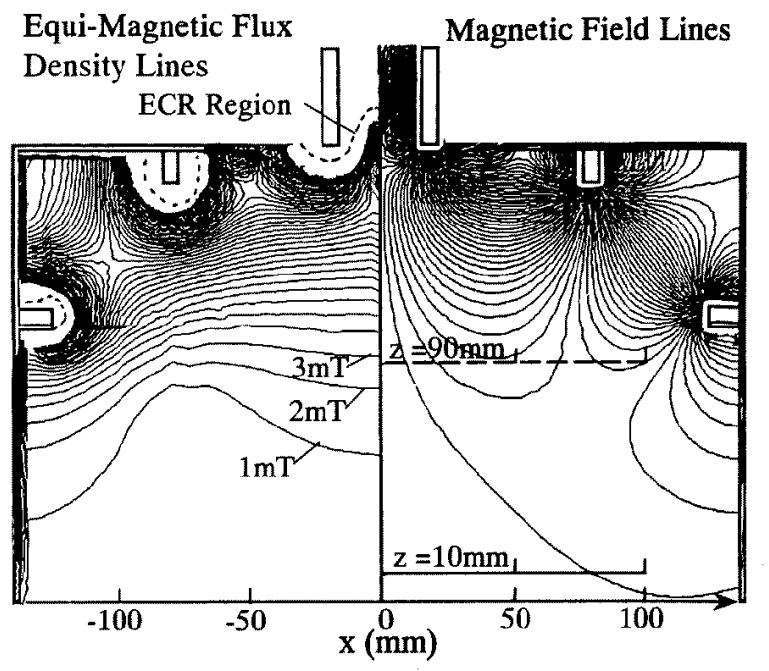

Fig.3. Calculated source magnetic field

vacuum chamber and the ion beams were not extracted. So, the source properties were investigated through the source plasma diagnostics with Langmuir probes. The probes were inserted through the slits shown in Fig.2(c) and swept along the slit direction. Plane probes of diameters $\phi 1.5, \phi 1.0$ or $\phi 0.8 \mathrm{~mm}$ were used to measure the spatial plasma parameter distributions. Electron energy distribution was measured with a cylindrical probe of $\phi 0.4 \times 6 \mathrm{~mm}$. The probe support was covered with a ceramic material. Co-ordinates $(x, y, z)$ and their starting points are shown in Fig.2.

\section{Experimental Results}

An ion source performance is estimated with the amount and qualities of extracted ion beams. For microfabrication use, ion source working pressure is appropriate as $2-6 \times 10^{-2} \mathrm{~Pa}$. With this gas pressure, ion beam current density of $1.0 \mathrm{~mA} / \mathrm{cm}^{2}$ is necessary. In this study, the beam was not extracted. So, Langmuir probes were used to measure ion saturation currents.

A $\phi 1.5 \mathrm{~mm}$ plane probe was used for the ion saturation current Ii measurements in Figs. 4 and 5 . The probe bias voltage was $-100 \mathrm{~V}$. The total ion current flowing into the measuring plate biased to $-30 \mathrm{~V}$ was measured. Above this bias voltage, the total ion current did not increase.

Fig.4 shows the measured ion saturation current distribution on the upper half of the plate. The ion saturation current varies below $\pm 5 \%$ within $180 \mathrm{~mm}$ along the $\mathrm{x}$-axis and $240 \mathrm{~mm}$ along the $\mathrm{y}$-axis. This result shows a uniform, large area plasma is generated near the measuring plate.

Fig.5 shows the dependence of the ion saturation current distribution on the gas pressure. The ion saturation current increases from the pressure of $1.3 \times$ $10^{-2}$ to $2.7 \times 10^{-2} \mathrm{~Pa}$ and then saturates. When the gas pressure increases above $4.0 \times 10^{-2} \mathrm{~Pa}$, the ion saturation current at the plate center becomes larger than at the circumference. So, the uniformity degrades when the gas pressure increases above $4.0 \times 10^{-2} \mathrm{~Pa}$. 

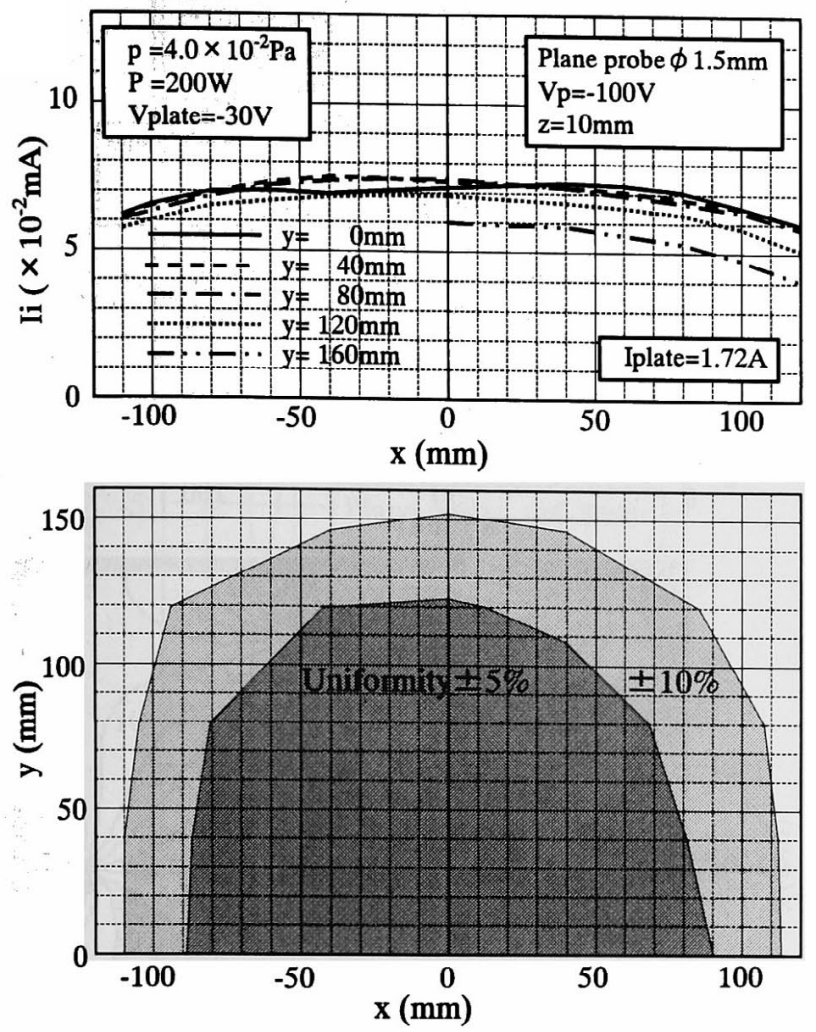

Fig.4. Ion saturation current distribution near measuring plate.

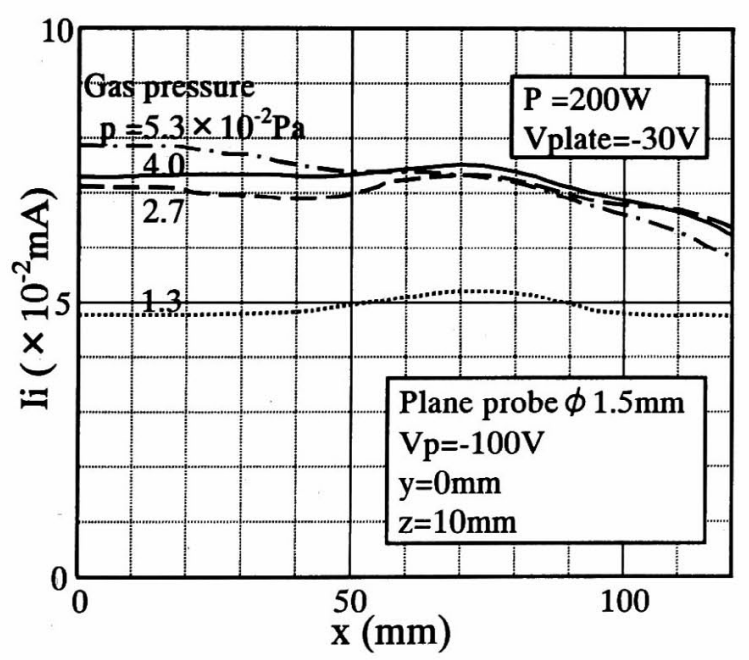

Fig.5. Ion saturation current distribution gas pressure dependency.

\section{Discussion}

To confirm the plasma generation scheme, the following items were investigated.

\subsection{Plasma generation in the field-free region}

- Plasma region movement dependence on gas pressure

As shown in Fig.5, the ion saturation current increases from $1.3 \times 10^{-2} \mathrm{~Pa}$ to $2.7 \times 10^{-2} \mathrm{~Pa}$ or more. The plasmagenerating region is expected to move with these gas pressures. To confirm the predicted plasma-generating region, ion saturation current distributions were examined at three points $(\mathrm{z}=90,110,130 \mathrm{~mm})$ and compared with those at the point $(\mathrm{z}=10 \mathrm{~mm})$ near the measuring plate. Figs. 6 and 7 show the results and the magnetic field distributions at the same time. Applied microwave power was 200 (W) each.

The ion saturation current becomes largest between two magnet poles $(x \fallingdotseq 45 \mathrm{~mm})$ in Fig. 6 with the low gas pressure; $1.3 \times 10^{-2} \mathrm{~Pa}$. The ion saturation current at $\mathrm{z}=90 \mathrm{~mm}$ shows a relatively uniform distribution, and its value is about $1 / 3$ of the peak value at $z=130 \mathrm{~mm}$. The main plasma-generating region is the shadowed region shown in Fig. 6 with the low pressure. The ion saturation current at $\mathrm{z}=90$ or $10 \mathrm{~mm}$ in Fig. 7 is 1.5 times larger than that in Fig.6. So, the results in Figs. 6 and 7 mean there is very little plasma diffusion from the strong magnetic field region (shadowed in Fig.6). The results also mean there is uniform and dense plasma generation in the magnetic field-free region (shadowed in Fig7), with the moderate gas pressure; $2.7 \times 10^{-2} \mathrm{~Pa}$. The plasma generation at the magnetic field-free region means easy plasma diffusion to the beam extraction region, and as a result, dense plasma is generated at the extraction electrode where the measuring plate is mounted.

\section{- Evaluation of generated ion amount and electron energy distribution in the magnetic field-free region}

The above results show there is dense plasma generation in the field-free region. Hot electrons to generate the plasma were measured and discussed as follows to confirm plasma generation in the magnetic field-free region. Fig.8 shows electron current characteristics (on a semi-logarithmic graph) measured with the cylindrical probe at $4.0 \times 10^{-2} \mathrm{~Pa}$ and microwave power of 200W. This graph shows hot electrons exist in addition to bulk plasma electrons. As for the bulk electrons, density ne $=3.5 \times 10^{10} \mathrm{~cm}^{-3}$ and temperature $\mathrm{Te}=4 \mathrm{eV}$ are obtained. According to these results and neutral particle density $\mathrm{n}_{0}=1.0 \times 10^{13} \mathrm{~cm}^{-3}$, ion generation rate in the field-free region was estimated as follows. As shown in Fig.9, plasma potential Vs was 9V. Ionizing potential of Ar was $16 \mathrm{~V}$. So, the hot electrons measured below $V p=-7 \mathrm{~V}$ are effective for ionization. Their temperature is $13 \mathrm{eV}$, and their density is estimated as $\mathrm{neh}=3.0 \times 10^{9} \mathrm{~cm}^{-3}$. From these values, ion generation rate per unit volume $Q$ is as follows.

$$
\begin{aligned}
\mathrm{Q} & =\text { neh } \cdot \mathrm{n}_{0} \cdot\langle\sigma \mathrm{V}\rangle \\
& =3.0 \times 10^{9} \cdot 1.0 \times 10^{13} \cdot 3.5 \times 10^{-8} \\
& =1.1 \times 10^{15} / \mathrm{s} / \mathrm{cm}^{3}
\end{aligned}
$$

Assuming that all ions generated within a $10 \mathrm{~cm}$ depth from the measuring plate flow into the plate, the estimated total ion current into the plate is $\mathrm{e} \times \mathrm{Q} \times$ measuring plate area $\times 10 \mathrm{~cm}$,i.e.,1.6A. This value agrees with the measured value,1.67A. The plasma density increases in the upper part in the discharge chamber as shown in Fig.7, on the other hand, some ions are lost at the wall where the magnetic field is weak. These two effects cancel each other, which means the above explanation is valid. 

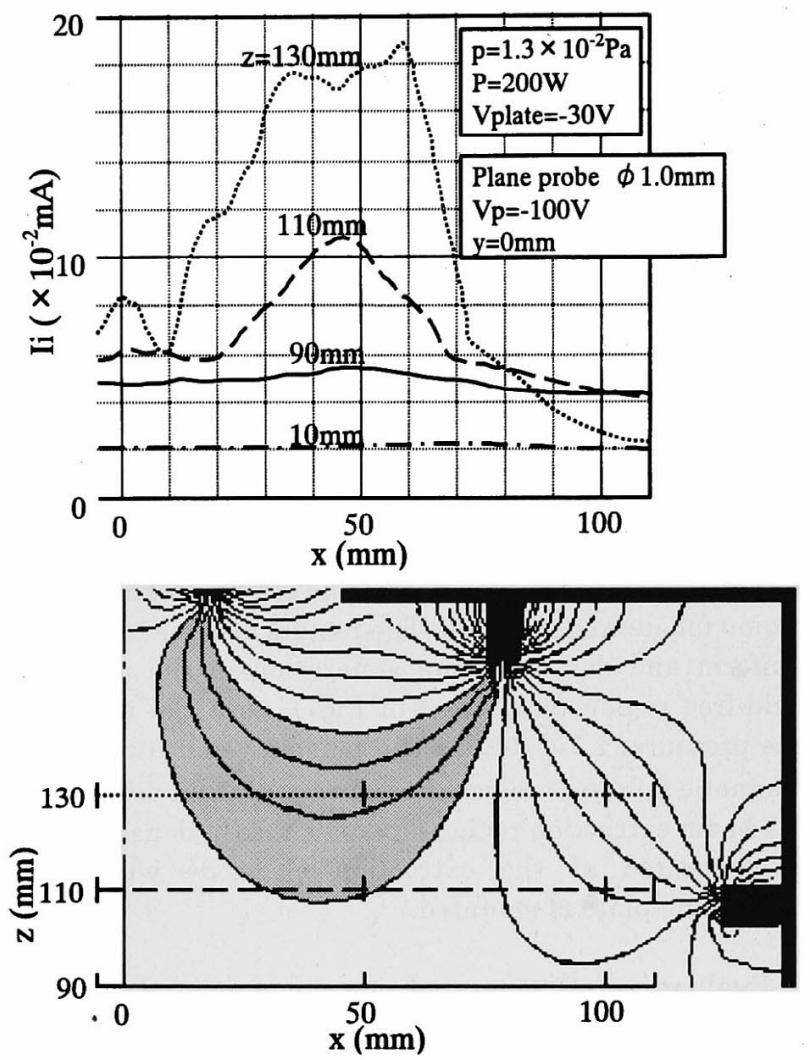

Fig.6. Ion saturation current sectional distribution $\left(1.3 \times 10^{-2} \mathrm{~Pa}\right)$

5.2 Hot electrons flowing into the magnetic field-free region

- Hot electrons flowing along magnetic field lines

In ref.(3), hot electrons flowing along magnetic field lines of multipolar magnetic field have been presumed from measured floating potential distribution dips. The results shown in 5.1 support plasma generation in the field-free region by hot electrons. Here, hot electrons flow was investigated by probe current distribution measurements, to confirm the hot electrons flowing into the magnetic field-free region.

Fig.9 shows the probe current Ip distribution with a $\phi$ $0.8 \mathrm{~mm}$ plane probe at the middle point $(\mathrm{z}=90 \mathrm{~mm})$, or at the lower point $(\mathrm{z}=10 \mathrm{~mm})$, shown in Fig.3, under the conditions: $4.0 \times 10^{-2} \mathrm{~Pa}$ gas pressure; microwave power $200 \mathrm{~W}$; and probe bias voltage $\mathrm{Vp}=-20 \mathrm{~V}$. As shown in Fig.9, the probe currents include the hot electrons over $29 \mathrm{eV}$, because the plasma potential $\mathrm{Vs}=9 \mathrm{~V}$ and the probe bias voltage $V p=-20 \mathrm{~V}$. The probe current distribution at the middle discharge chamber $(\mathrm{z}=90 \mathrm{~mm})$ has a sharper peak at the center compared to the lower region $(z=10 \mathrm{~mm})$. The measuring plane of this probe crosses the magnetic field lines normally, so, the measured hot electrons flow along the magnetic field lines. The current distribution by the cylindrical probe measurements has no sharp peak, contrary to the plane probe measurements shown in Fig.9. The results in Fig.9 mean the divergence of hot electrons occurs along magnetic field lines at the magnetic field-free region.
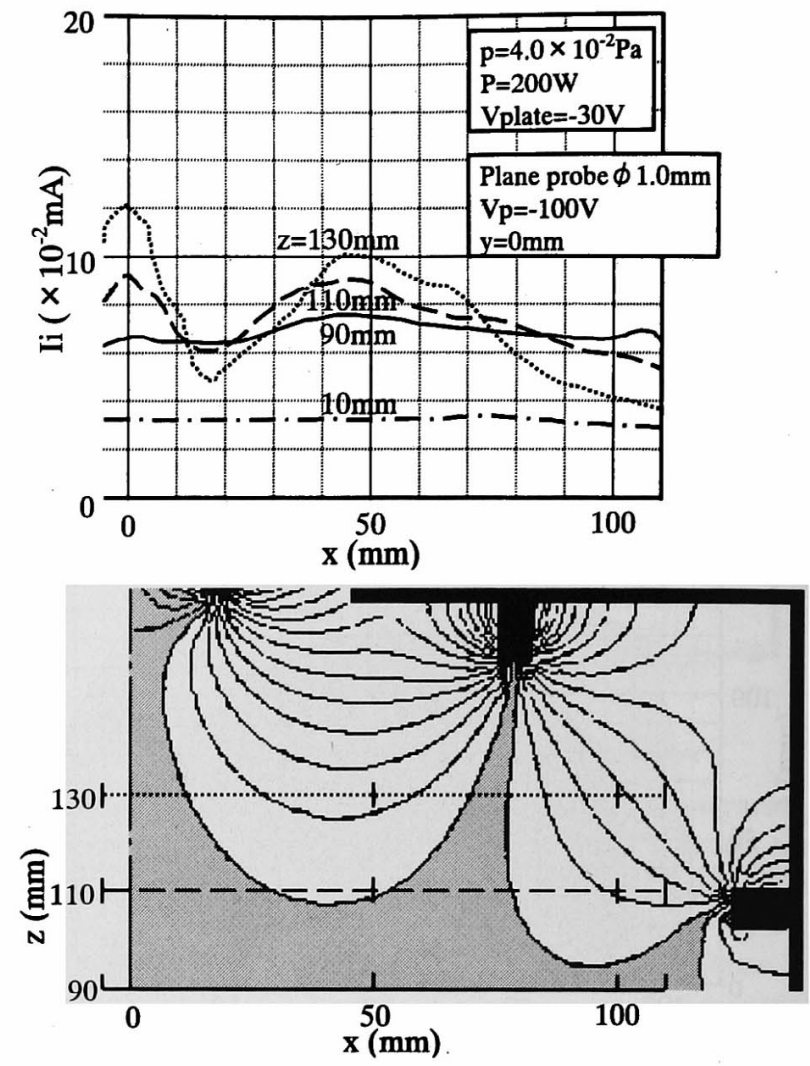

Fig.7. Ion saturation current sectional distribution $\left(4.0 \times 10^{-2} \mathrm{~Pa}\right)$

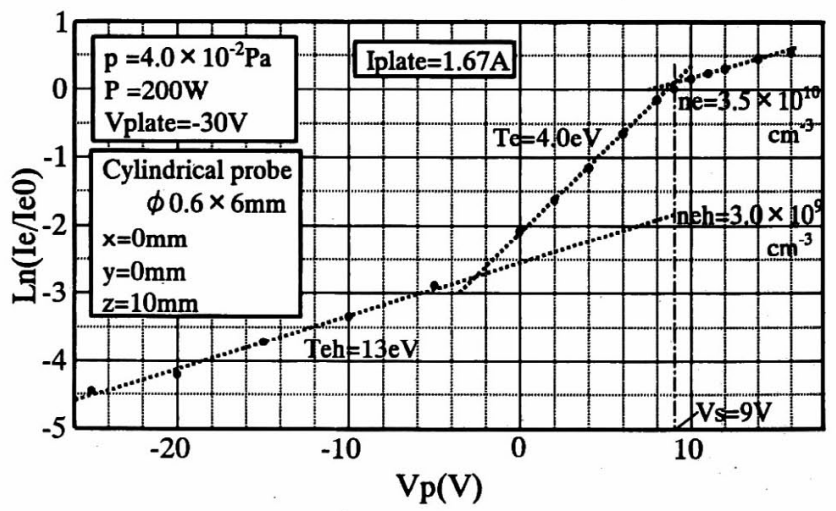

Fig.8. Electron current characteristics measured with cylindrical probe

- Estimation of hot electrons amount flowing into the field-free region

Fig.10 shows the probe current density Jp distribution dependency on the probe bias voltage Vp under the same microwave power as Fig.9. The probe was a $\phi 1.5 \mathrm{~mm}$ plane type. The probe current under the deep bias voltage means ion current. By decreasing the bias voltage, the probe current includes the high energy electron current over the negative potential bias.

From Fig.8, the plasma potential Vs was $9 \mathrm{~V}$ at the place in Fig. 10 and Vp was -20V. So, the hot electrons with over $29 \mathrm{eV}$ energy flow into the probe. Assuming the ion current is given by the dashed lines of Fig.10, the hot electrons $(>29 \mathrm{eV})$ current has the peak value of $6 \mathrm{~mA} / \mathrm{cm}^{2}$ 


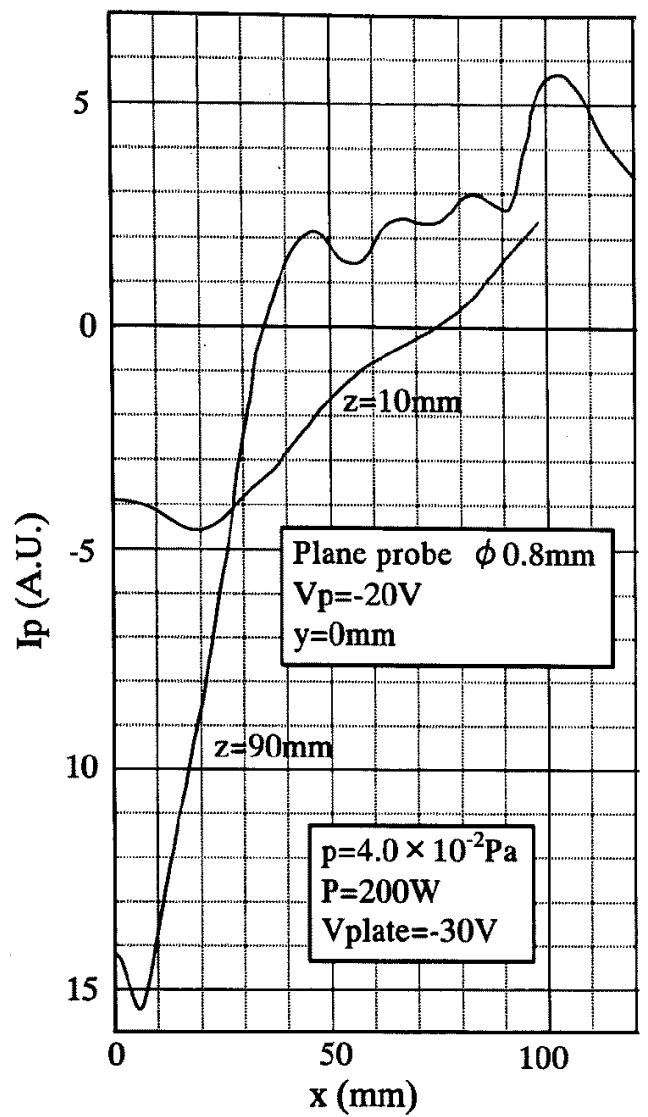

Fig.9. Compared hot electron current distributions

and decreases linearly from the center. The average electron current density over $29 \mathrm{eV}$ is about $2 \mathrm{~mA} / \mathrm{cm}^{2}$ in a $22 \times 28 \mathrm{~cm}^{2}$ area. So, the total electron current over $29 \mathrm{eV}$ is estimated to be 1.2A. By increasing the probe bias voltage with $10 \mathrm{~V}$ more lowly $(\mathrm{Vp}=-30 \mathrm{~V})$, the electron current becomes a half of the current at $V p=-20 \mathrm{~V}$. When $\mathrm{Vp}=$ $50 \mathrm{~V}$ is applied, the electron current decreases to $1 / 8$ compared to the value at $\mathrm{Vp}=-20 \mathrm{~V}$. These results show the probe current decreases exponentially with the probe bias voltage. The electron current measured by the probe is the high energy $(>29 \mathrm{eV}$ ) component of the total electron current $10 \mathrm{~A}$ and temperature $14 \mathrm{eV}$. Assuming the energy distribution as Maxwellian, the current Ie flowing into the probe is $\mathrm{Ie} / \mathrm{Ieo}=\exp [\mathrm{e} \cdot(\mathrm{Vp}-\mathrm{Vs}) / \mathrm{kTe}]$. Here $\mathrm{Vs}=9 \mathrm{~V}$ (Fig.8) and $\mathrm{Ie}, \mathrm{Vp}$ are measured, then Ieo=10A and $\mathrm{kTe}=14 \mathrm{eV}$ are obtained. The value $14 \mathrm{eV}$ is nearly equal to the measured value $13 \mathrm{eV}$ of the hot electrons obtained by the cylindrical probe as shown in Fig.8. The hot electrons measured by the cylindrical probe seem to be thermalized electrons flowing along magnetic field lines into the magnetic field-free region.

5.3 The total power consumption to produce hot electrons

The amount of hot electrons was investigated from the viewpoint of the power consumption.

The hot electrons flowing into the magnetic field-free region are heated by microwave power at the heating region near the microwave window where the strong

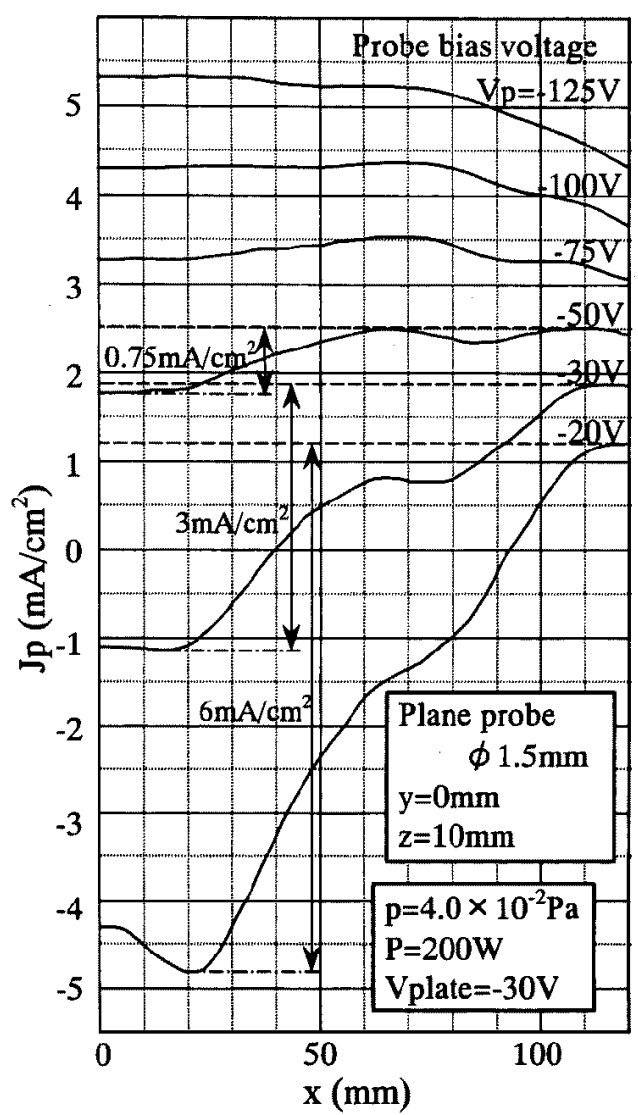

Fig.10. Hot electron current distributions with probe bias voltages

magnetic field is applied for the ECR condition.

Assuming that the electrons, flowing into the heating region, are bulk ones with $4 \mathrm{eV}$ temperature in the magnetic field-free region, and that they are then heated to $14 \mathrm{eV}$ after flowing out from the heating region, the power $\mathrm{Pe}$ consumed at the heating region is $\mathrm{IeO} / \mathrm{e} \cdot \Delta$ $E=10(\mathrm{~A}) / \mathrm{e} \times[14-4(\mathrm{eV})]=100 \mathrm{~W}$. This $\mathrm{Pe}$ is half of the input microwave power $200 \mathrm{~W}$. The residual power will be consumed at the plasma near the strong cusp magnetic field and the wall.

The amount of the hot electrons current $I e 0=10 \mathrm{~A}$ is explained as follows. The hot electrons are heated at the heating region with ECR condition. The area of ECR AECR is calculated from the constant total magnetic flux law ; $\mathrm{A}_{\mathrm{ECR}} \cdot \mathrm{B}_{\mathrm{ECR}}=\mathrm{A} \cdot \mathrm{B}$. Here, $\mathrm{B}_{\mathrm{ECR}}=87.5 \mathrm{mT}, \mathrm{B}=3 \mathrm{mT}$, and $A=200 \mathrm{~cm}^{2}$. Therefore, $A E C R=7 \mathrm{~cm}^{2}$. Assuming the electron energy distribution as Maxwellian, the electron current density je is calculated from the electron density ne and the temperature $\mathrm{Te}$ as follows.

je $=\mathrm{Ie} / \mathrm{A}=\mathrm{e} \cdot \mathrm{ne} \cdot \mathrm{ve} / 4$

Here, ve $=\sqrt{ }(8 \mathrm{kTe} / \pi \mathrm{me})$

ve: electron velocity, me : electron mass Therefore, to explain $\mathrm{Ie}_{0}=10 \mathrm{~A}$ with $\mathrm{AECR}_{\mathrm{E}}=7 \mathrm{~cm}^{2}, \mathrm{kTe}=4 \mathrm{eV}$, the electron density ne at $\mathrm{ECR}$ region is $3 \times 10^{11} \mathrm{~cm}^{-3}$. This value is about 10 times larger than the measured value in the lower part $(\mathrm{z}=10 \mathrm{~mm})$. But, Fig.7 shows a gradual electron density increase along the discharge chamber's center line from the lower part to the upper ECR region. 
So, the result of 10 times larger electron density than in the lower part is considerable.

\section{Summary}

An experimental ion source (inner sizes of the discharge chamber: length $360 \mathrm{~mm}$, width $270 \mathrm{~mm}$, height $170 \mathrm{~mm}$ ) was constructed. Microwave power was introduced at a local point in the discharge chamber to produce hot electrons. Uniform, large area plasma was generated by hot electron collisions with the gas in the magnetic fieldfree (below $3 \mathrm{mT}$ ) region surrounded by a magnetic multipolar field. The performance of the source plasma obtained for argon was as follows:

1. Plasma uniform area was $240 \times 180 \mathrm{~mm}$ (uniformity $\pm 5 \%$ ). Average ion current density was $1.75 \mathrm{~mA} / \mathrm{cm}^{2}$.

(Conditions: gas pressure $4 \times 10^{-2} \mathrm{~Pa}$, microwave power $200 \mathrm{~W}$ )

2. Plasma production in the field-free region was confirmed above the pressure $2.7 \times 10^{-2} \mathrm{~Pa}$.

(Manuscript received Apr. 7, 1999, revised Oct. 6, 1999)

References

(1) M. Pichot, A. Durandet, "Microwave multipolar plasmas excited by distributed electron cyclotron resonance: Concept and performance", Rev. Sci. Instrum. 59, pp.1072-1075(1988)

(2) G. Neumann, K.H.Krettschmer," Characterization of a new electron cyclotron resonance source working with permanent magnets", J. Vac. Sci. Technol., B9, pp.334-338(1991)

(3) N. Sato, S. lizuka, Y. Nakagawa, T. Tsukada, "Uniform plasma produced by a plane slotted antenna with magnets for electron cyclotron resonance", Appl. Phys. Lett., 62, pp.1469-1471(1993)

(4) N. Shida, T. Inoue, H. Kokai, Y. Sakamoto, W. Miyazawa, S. Den, Y. Hayashi, "Large-Area Electron Cyclotron Resonance Plasma Source with Permanent Magnets", Jpn. J. Appl. Phys., 32, pp.L1635L1637(1993)

S. Ichimura (Member) was born in 1967. He received his

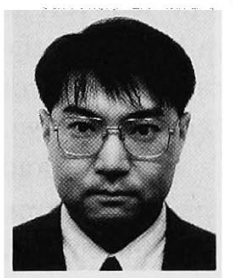
B.E. and M.E. degrees in Aeronautics from University of Tokyo in 1990 and 1992, respectively. He joined Hitachi Ltd. in 1992. His research interests include large area ion source developments.

T. Sato (Member) was born in 1944. He received his

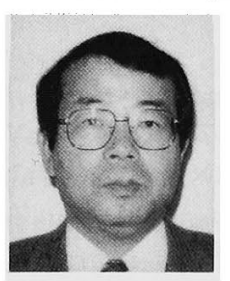
B.E. and D.E. degrees in Electrical Engineering from University of Tokyo in 1967 and 1992, respectively. He joined Hitachi Ltd. in 1967. His research interests include large area ion or electron source developments.
S. Ooishi (Non-member) was born in 1944. He

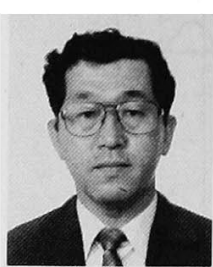
graduated from Shizuoka Technical High School in 1963. He joined Hitachi Ltd. in 1963. He works on design and development of large area ion source. 